\title{
Combinando Impresión 3D y electrónica como estrategia para mejorar la experiencia de aprendizaje
}

\section{(Combining $3 \mathrm{D}$ printing and electronics as a strategy for improving the learning experience)}

\author{
Juan Suardíaz Muro \\ Mario Pérez Gomáriz \\ Andrés Cabrera Lozoya \\ René Ove Do Carmo Trolle \\ Universidad Politécnica de Cartagena, UPCT (España)
}

DOI: http://dx.doi.org/10.5944/ried.24.1.27596

\section{Cómo referenciar este artículo:}

Suardíaz Muro, J., Pérez Gomáriz, M., Cabrera Lozoya, A., y Do Carmo Trolle, R. O., V. (2021). Combinando Impresión 3D y electrónica como estrategia para mejorar la experiencia de aprendizaje. RIED. Revista Iberoamericana de Educación a Distancia, 24(1), pp.115-135. doi: http://dx.doi.org/10.5944/ried.24.1.27596

\section{Resumen}

En la actualidad, la impresión 3D se ha convertido en una tecnología que ha creado un nuevo paradigma de comunidades de aprendizaje, constituyendo una cultura o subcultura contemporánea, denominada coloquialmente como "cultura maker", la cual puede considerarse como una extensión de la cultura DIY (Do It Yourself o hágalo usted mismo). Se trata de una nueva cultura basada en la tecnología y en el uso de herramientas que ha tenido gran aceptación en campos diversos como la ingeniería, medicina, arquitectura y artesanía. El presente artículo muestra una experiencia de integración de aprendizaje de Impresión 3D con electrónica dentro del currículo de la titulación de Ingeniería Industrial, especialidad en Mecánica. Para ello se ha utilizado la metodología de Aprendizaje Basado en Servicio. Se ha localizado una posible necesidad social y, a partir de esta necesidad se ha propuesto el diseño de una solución por parte de los alumnos. El ejemplo seleccionado ha sido el desarrollo de una mano robótica capaz de comunicarse mediante el lenguaje de signos. Con este trabajo los alumnos han reforzado sus conocimientos de modelado 3D utilizando el software de modelado Solid Works, así como sus conocimientos de electrónica, teniendo que desarrollar el sistema de control de los motores de las articulaciones de la mano usando un sistema basado en el popular microcontrolador Arduino. 
Palabras clave: impresión 3D; arduino; aprendizaje basado en servicio; electrónica; robótica.

\begin{abstract}
Today, $3 \mathrm{D}$ printing has become a technology that has created a new paradigm of learning communities, constituting a contemporary culture or subculture, colloquially known as "maker culture", which can be considered an extension of DIY (Do It Yourself) culture. It is a new culture based on technology and the use of tools that has been widely accepted in diverse fields such as medicine, architecture and handicraft. This article shows an experience of integration of learning of $3 \mathrm{D}$ printing with electronics within the curriculum of the Industrial Engineering degree, specialty in Mechanics. The methodology of Service Based Learning has been used for this purpose. A possible social need has been identified and, based on this need, the design of a solution by the students has been proposed. The selected example has been the development of a robotic hand capable of communicating through sign language. With this work the students have reinforced their knowledge of 3D modelling using the software Solid Works, as well as their knowledge of electronics, having to develop the control system for the motors of the hand's joints using a system based on the popular microcontroller Arduino.
\end{abstract}

Keywords: 3D printing; arduino; service based learning; electronics; robotics.

Las impresoras 3D son dispositivos capaces de crear objetos tridimensionales a partir de un modelo diseñado con ordenador mediante programas CAD (Computer Aided Design o diseño asistido por computadora).

El origen histórico de esta tecnología se puede considerar que se remonta a 1984. Es en este año cuando, tomando como base la tecnología desarrollada en 1976 de impresión por inyección de tinta (2D), se inician las primeras experiencias orientadas a realizar impresión tridimensional. Se atribuye la invención de la tecnología a Charles Hull, quien marcó el germen de inicio con el desarrollo de la patente denominada "Apparatus for production of three-dimensional objects by stereolithography” (Hull, 1984) y fundó la empresa 3D Systems.

Escobar (2018) marca como hitos más relevantes en la evolución histórica de esta tecnología los siguientes:

- 1992. Fabricación de prototipos capa por capa, realizados por la empresa 3D Systems, donde un fotopolímero que se va depositando es endurecido mediante luz ultravioleta.

- 1999. Nuevos avances en medicina al crear órganos artificiales mediante impresión 3D. Se trata de la primera aplicación médica de esta tecnología, en la que científicos del Instituto de Wake Forest de Medicina Regenerativa diseñan con impresión $3 \mathrm{D}$ una vejiga artificial que se recubre con células del paciente con objeto de evitar el rechazo. 
- 2002. Un riñón 3D plenamente funcional, realizado también por científicos del Instituto de Wake Forest de Medicina Regenerativa.

- 2005. Open-source colabora con la impresión 3D. Se produce un punto de inflexión en el avance de esta tecnología cuando surge la iniciativa de la creación y lanzamiento de ReRaP, un proyecto de código abierto con la finalidad de crear una impresora capaz de imprimir la mayoría de sus propios componentes.

- 2006. El SLS y la personalización en la fabricación en masa. SLS se corresponde con las siglas de Sintetización por Láser Selectivo, un proceso por el cual un láser va fundiendo el material usado para la impresión. Durante este año también se produjo una máquina que permitía imprimir en múltiples materiales, entre los que se incluyen polímeros y elastómeros.

- 2008. Aparece la primera impresora con capacidad de auto réplica. Como resultado de la iniciativa ReRap surge Darwin, la primera impresora que es capaz de imprimir la mayoría de sus componentes. De esta forma un usuario podría reparar componentes de su propia impresora y usar una para fabricar otras.

- 2008. Shapeways lanza una página que permite compartir diseños en $3 \mathrm{D}$ como objetos físicos de bajo coste a artistas, arquitectos y diseñadores. Se crea así una comunidad de usuarios de impresoras que intercambian diseños e ideas, acelerando su inclusión en el mercado.

- 2008. Presentación de la primera persona caminando sobre una prótesis de pierna impresa en 3D sin necesitar ningún tipo de montaje.

- 2009. Los kits de impresoras 3D DIY entran en el mercado. La empresa MakerBot introduce los primeros kits de impresoras para montarse uno mismo.

- 2011. Primer avión impreso en 3D, construido en la universidad de Southampton. Se trataba de un modelo no tripulado, impreso durante 7 días y con un coste asociado de 7.000 euros.

- 2011. Primer coche impreso en 3D. Inspirado por reducir peso y aumentar la eficiencia energética, la empresa Kor Ecologic presenta Urbee, un prototipo de coche en el que toda su carrocería fue diseñada e impresa en 3D.

Gracias a toda esta evolución y demostración de capacidades, a lo largo de los últimos años, la impresión $3 \mathrm{D}$ ha despertado un interés con un crecimiento exponencial. Una noticia publicada por la plataforma Universia en diciembre de 2019 (Universia, 2019) considera la figura del impresor 3D como uno de los trabajos del futuro, ya que esta tecnología ha creado una serie de nichos de mercado importantes para profesionales formados en esta técnica. Según resalta esta plataforma, algunos de los sectores más afines para el uso de esta tecnología son:

- Ingeniería: donde se podrá utilizar la impresión de piezas en la reposición o sustitución en tareas de mantenimiento, o en el diseño de maquetas o prototipos.

- Arquitectura: permitiendo a los profesionales crear maquetas que aporten valor añadido, o incluso a usarla como material de construcción, habiendo ya algunas 
iniciativas, aún incipientes, en lo que se refiere a construcción de edificios utilizando enormes impresoras 3D que proyectan hormigón.

- Medicina: posibilita a doctores, veterinarios u odontólogos la impresión de prótesis a medida y donde hay una línea de investigación puntera en lo referente a la impresión de órganos utilizando materiales biocompatibles.

- Arte y Moda: presentando un uso destacado de impresoras $3 \mathrm{D}$ en el diseño de calzados, joyas, prendas, etc.

- Investigación Espacial: en vez de tener que trasladar al espacio, con el coste adicional de combustible asociado al excedente de peso que ello supone, un repertorio extenso de piezas de repuesto, la NASA está trabajando actualmente en iniciativas que usen este tipo de impresión para crear en el propio espacio el repuesto que sea necesario en cada momento.

Son varias las iniciativas dedicadas a combinar el uso de la impresión 3D en el campo de la innovación educativa, tanto a nivel de educación pre-universitaria como universitaria (Bosqué 2015; Bull et al., 2015; Beltrán y Rodríguez, 2017; Moeck et al., 2019). Así, por ejemplo, en Dahle (2018) y Dahle et al. (2019) se presentan varias iniciativas en las que se utiliza esta tecnología en la docencia sobre fabricación y simulación de dispositivos MEMs. En el campo de la robótica, resulta una buena combinación en asignaturas en las que se quieran practicar de forma conjunta conceptos de diseño mecánico, programación y electrónica, utilizándose para diseñar robots a medida que los estudiantes diseñan y controlan (González-Gómez et al., 2012; Yu et al., 2017; Moreno et al., 2016). Domínguez et al. (2013) describen los beneficios de usarla en la docencia en arquitectura, donde los alumnos pueden plasmar y pasar del plano a la realidad las construcciones que diseñan. Dentro de este campo, Buswell et al. (2018) presentan un sistema en el que se fabrica una impresora 3D de gran tamaño, capaz de usar cemento como material de fabricación, sentando así las bases de impresoras robóticas capaces de fabricar edificios u otro tipo de estructuras civiles como puentes, si bien es un campo en el que aún queda camino por recorrer. También se han propuesto iniciativas docentes con el objeto de crear nuevas formas de potenciar y desarrollar la creatividad en el ámbito de las escuelas de Arte (Bonet et al., 2017) y en aplicaciones de diseño industrial (Bull et al., 2015). Otro ámbito de aplicación en la que también está obteniendo buenos resultados es el campo de la química (Pinger, Geiger y Spence, 2019; Pernaa y Wiedmer, 2019), donde se utiliza para imprimir las moléculas o los átomos que constituyen diferentes compuestos y poder entender la disposición de sus enlaces u orbitales moleculares. También hay experiencias en el campo de la cristalografía, con objeto de reproducir a escala modelos cristalográficos asociados al crecimiento de diferentes tipos de minerales (Brannon et al., 2020; Murrey 2019). Uno de los ámbitos en los que cada vez se encuentra más integrada es en docencia de la medicina (Heinze et al., 2020; Osakwe et al., 2019; Reymus et al., 2019; Mullan y Carter, 2020), pues no hay que olvidar que, tal y como se ha comentado anteriormente, varios de los hitos asociados 
a su evolución histórica han estado muy unidos a desarrollos y aplicaciones en este campo. En el campo de la Geología y Paleontología se ha utilizado para mejorar el aprendizaje sobre conceptos asociados a terremotos (Kyriakopoulos, 2019) o para enseñar sobre procedimientos de fosilización (Johnson y Carter, 2019; Peterson y Krippner, 2019). La Biología también se ha beneficiado, aplicándolo a representación de células, esqueletos de vertebrados, reproducción de partes de plantas o animales (Hansen et al., 2020; Benjamin, 2020). Incluso un campo tan abstracto como es el de las matemáticas no ha escapado a iniciativas docentes que utilizan esta tecnología. Así, Dilling y Witzke (2020) describen una iniciativa donde la impresión 3D es utilizada para asentar conocimientos sobre derivadas y funciones; $\mathrm{u}$ otras similares, como la de Reichenberger et al. (2019), orientada al aprendizaje matemático de poliedros y superficies.

En cuanto al proceso de formación y aprendizaje del uso de esta tecnología, si bien hay ciertas dificultades para conseguir un nivel de aprendizaje avanzado, para alcanzar un nivel básico en el que se puedan desarrollar los primeros prototipos no se requiere una curva de aprendizaje excesivamente larga.

A modo de resumen, el proceso de formación requerido para capacitarse en esta tecnología requeriría de las siguientes fases:

1. Introducción a la Fabricación Aditiva. En esta fase se aprenderían las bases de lo que sería la fabricación aditiva, su concepto y los materiales utilizados. Se estudiarían los diferentes tipos de impresoras, así como los tipos de filamentos y materiales de los que están hechos. En la actualidad, existen diferentes tipos de filamentos, permitiendo imprimir con apariencia de madera, piedra, bronce y hasta es posible imprimir chocolates y golosinas.

2. Aprendizaje de un software para el modelado tridimensional. Para poder imprimir un objeto 3D antes hay que definir, crear y modelar el objeto. Para ello existen diversos programas, unos gratuitos y otros comerciales, que permiten realizar esta labor. El objetivo final es generar un fichero STL. No está muy claro el verdadero significado de esta extensión. El más aceptado es STereoLitograpy, debido a que fue esta tecnología la primera en adoptar el formato. Otros significados comunes son el de "Standard Triangle Language" o "Standard Tessellation Language". Se trata de un formato de diseño asistido por computadora (CAD), de tipo binario o ASCII, que define la geometría de objetos en 3D, excluyendo información como el color, las texturas y otras propiedades físicas que sí incluyen otros formatos CAD. La principal ventaja de esta fase es que en muchas escuelas de ingeniería ya se enseña software de dibujo y modelado, por lo que el alumno habitualmente ya tiene esta competencia adquirida y solamente hace falta explicarle cómo generar el fichero con extensión STL a partir del software de modelado al que esté habituado a utilizar.

3. Para imprimir un objeto $3 \mathrm{D}$, la fabricación aditiva lo que hace es "cortar" el objeto en capas o rodajas (slices en inglés) y la impresora, partiendo de la base 
del objeto, va creando cada capa depositando el material de filamento que sale fundido por la denominada boquilla extrusionadora de la impresora. Para que el dispositivo pueda realizar esta tarea, es necesario un programa adicional, denominado 'slicer', que toma como entrada el fichero STL y va generando las diferentes capas que la impresora va creando durante el proceso de impresión. El resultado del 'slicer' es un fichero denominado G-Code que genera información útil para la impresora, que sólo entiende de datos asociados a movimientos a determinadas coordenadas tridimensionales $\mathrm{X}, \mathrm{Y}, \mathrm{Z}$ y expulsar cierta cantidad de material en esa posición. Es decir, para cada una de las capas o rodajas creadas, este software calcula los movimientos que debe hacer el cabezal para rellenar cada área. En cierta forma, el 'slicer' es el equivalente del programa CAM (Computer Aided Manufacturing) de las máquinas CNC tradicionales.

Para realizar su trabajo, el 'slicer'tiene en cuenta una gran cantidad de parámetros: velocidad, temperatura, los parámetros de la máquina, dimensiones máximas, y de material. Si bien, lo habitual es que vengan cargados una serie de valores por defecto que permiten poder realizar una impresión satisfactoria sin excesiva dificultad, saber entender y configurar de forma experta estos parámetros no es tarea trivial y, de forma similar a los antiguos artesanos, cuanta más experiencia y pericia tenga el diseñador en el uso de estos parámetros, mejores saldrán las calidades y resultados de las impresiones finales.

4. Finalmente, el fichero G-Code se carga en la impresora 3D, encargada de realizar el proceso final de impresión. Para ello, la impresora toma el filamento, enrollado en un carrete ubicado en su extremo superior, y lo va derritiendo en el cabezal extrusor a una temperatura de entre $180^{\circ}$ y 220 grados. Con el plástico fundido, la máquina crea una capa; se eleva unas décimas de milímetro y pinta una nueva capa, continuando de forma sucesiva hasta finalizar la impresión del objeto de acuerdo con el plano inicial. Para realizar esta serie de movimientos, la impresora dispone de una serie de motores y tornillos sin fin. En este proceso también hay que configurar una gran cantidad de parámetros, como las velocidades a las que debe llegar el cabezal en cada punto o las temperaturas a la que se debe calentar el filamento, entre otros. Una vez más, es habitual que el software establezca una serie de valores por defecto a estos parámetros y comprender y dominar la totalidad de estos parámetros de configuración requiere tiempo y es muy dependiente del modelo de impresora utilizado.

No es de extrañar entonces que una formación en este tipo de tecnología sea de interés para el futuro ingeniero. El caso aquí expuesto se corresponde con la docencia reglada en la titulación de Grado en Ingeniería Mecánica. El currículo asociado presenta una asignatura de Expresión Gráfica, donde se imparten conocimientos de modelado en 3D utilizando el entorno de desarrollo Solid Works y una asignatura de Fundamentos de Electrónica Industrial, donde se imparten conocimientos de electrónica analógica y digital. La experiencia se enmarca en el desarrollo de un 
trabajo conjunto, realizado de forma colaborativa con un grupo de 3 compañeros, donde se trabajan y refuerzan los conocimientos adquiridos en ambas asignaturas. Para ello, se ha utilizado la metodología de Aprendizaje Basado en Servicio.

\section{MARCO DE LA EXPERIENCIA}

Esta experiencia se desarrolla tratando de combinar el uso de nuevas tecnologías incipiente con la metodología de Aprendizaje por Servicio (ApS), la cual surgió en 1903 en la Universidad de Cincinnati como un movimiento de educación cooperativa, donde el trabajo, el servicio y el aprendizaje se integran de manera conectada. Las bases intelectuales de esta metodología fueron planteadas por John Dewey en el año 1905, aunque en ese momento no se definía directamente con este término (Arratia Figueroa, 2008).

El término ApS se incorpora en el año 1966 cuando se utiliza la frase "aprendizaje por servicio" para definir esta metodología que incluye a estudiantes, facultades y organizaciones. También, a finales de los años setenta, Robert Sigmon publicó los "tres principios del aprendizaje de servicio": los que reciben el servicio controlan el servicio prestado, los que reciben el servicio se vuelven más capaces de servir y ser servidos por sus propias acciones, y los que sirven son también sujetos que aprenden y que tienen un control significativo sobre lo que se espera que aprendan (Butin, 2003).

Actualmente, como afirma Roser Batle - una de las principales figuras de referencia de la metodología de aprendizaje del servicio en España - la mejor definición de Aprendizaje - Servicio podría ser la siguiente: "El aprendizaje-servicio es una propuesta educativa que combina los procesos de aprendizaje y el servicio a la comunidad en un único proyecto bien articulado, en el que los participantes se forman involucrándose en las necesidades reales del entorno para mejorarlo" (Batle, 2011, p. 2).

Se trata, pues, de una metodología orientada a la educación para la ciudadanía, inspirada en pedagogías activas y compatible con otras estrategias educativas. El ApS es un método para unir el éxito escolar y el compromiso social: aprender a ser competente y a la vez útil a los demás. Este método permite promover los siguientes tipos de aprendizaje:

- Competencias básicas: activa el ejercicio de todas las competencias del currículo, con énfasis en la competencia social y cívica y la iniciativa y autonomía personal.

- Valores y actitudes prosociales: estimula el esfuerzo, la responsabilidad y el compromiso solidario.

- Habilidades para la vida: Fortalece las habilidades psicosociales y la capacidad de participar en la vida social de manera positiva. 
Según Lucas Mangas (2012) y Lucas Mangas y Martínez (2012), los requisitos básicos para la ejecución de proyectos de Aprendizaje de Servicio son:

1. El aprendizaje debe estar asociado al servicio que se pretende prestar, y debe explicar lo que sus protagonistas aprenderán antes, durante y después de su realización.

2. El aprendizaje ayuda a comprender la realidad, a diagnosticar las necesidades, a realizar un servicio de calidad, a revisar la propia acción y a mejorarla en el futuro.

3. El servicio debe ser auténtico y responder a las necesidades reales del entorno con el fin de mejorarlo.

4. El proyecto debe ser educativo, planificado y evaluado por el educador, con una clara intención pedagógica.

5. La participación activa de los alumnos. Sus protagonistas deben ser los que intervengan en las diferentes fases del proyecto, desde la detección de necesidades hasta el diseño de propuestas de mejora.

Por otra parte, durante los últimos años, la nueva tecnología de impresión $3 \mathrm{D}$, combinada con desarrollos electrónicos de bajo coste, se han constituido como piezas claves de lo que se denomina "cultura Maker". Como beneficios de esta cultura se podrían destacar (Head, 2019):

- Permiten la inclusión digital al transformar a los participantes como productores, no sólo como consumidores.

- Fomentan interés por disciplinas STEAM (Science, Technology, Engineering, Arts, Maths); adquiriendo así competencias que son clave para la participación en una economía basada en la innovación tecnológica.

- Impulsan la creatividad y la generación de soluciones innovadoras.

- Promueven la exploración como vehículo para el aprendizaje por descubrimiento.

- Permiten el desarrollo de algunas de las competencias denominadas del siglo 21 (cognitivas, intrapersonales e interpersonales).

Debido a estos factores, la combinación de estas tecnologías en el aula podría considerarse como una forma de reforzar el aprendizaje en el aula. A este respecto, Cobo y Moravec (2011), frente al tradicional uso pasivo de la tecnología para aprender, abogan por una aproximación a la tecnología realizada de forma pragmática, de uso intencionado y con la finalidad última de mejorar la experiencia de aprendizaje.

Como objetivo de esta experiencia, se trata de combinar la metodología de aprendizaje servicio con un desarrollo práctico utilizando tecnologías novedosas como la impresión 3D, que supongan un reto y un desafío para los alumnos, de manera que puedan trabajar de forma colaborativa en la resolución del problema. 


\section{METODOLOGÍA}

El cuatrimestre se encuentra dividido en 15 semanas, dedicando 4 horas por semana a cada asignatura.

La estrategia seguida fue realizar 15 sesiones grupales (1 por semana), estructuradas de la siguiente forma:

- Sesión 01: Presentación del proyecto. En esta sesión se hace una descripción de la necesidad encontrada y se justifica su interés social. Para ello, se invita a un miembro de la asociación de personas sordas de Cartagena (ASORCAR) para que imparta una charla sobre la problemática.

- Sesión 02: Durante la primera semana, se hace una generación de grupos de 3 personas. En la experiencia participaron 21 alumnos, por lo que se constituyeron 7 grupos diferentes. Estos grupos se asignaron de forma aleatoria ya que se pretende hacer una simulación de un entorno laboral en el que el integrante tiene nuevos compañeros, de forma que también durante la realización del trabajo colaborativo los estudiantes practiquen sus competencias sociales. Se dedica esta sesión para hacer una dinámica de grupo para que los grupos se conozcan y se les plantea como trabajo para esa semana y la siguiente el desarrollo de una labor de búsqueda de información sobre posibilidades de diseño de manos biónicas.

- Sesión 03: Se hace un seguimiento de lo encontrado, se pone en común con el resto de grupos posibles ideas y se pide que hagan una planificación y estructura de trabajo para el diseño de una mano robótica que pueda realizar lenguaje de signos. Para ello se fija la siguiente estructura: deben dedicar 3 semanas para el modelado de la posible solución. Después de esa semana, se pondrán en común las soluciones propuestas y entre todos se elegirá una, la cual será fabricada y programada de forma conjunta durante las siguientes semanas.

- Sesiones 04 a 07: Cada grupo de estudiantes modela su propia solución.

- Sesión 08: Se presentan los modelos desarrollados y cada alumno va votando a una opción que no puede ser la propia. Se toma la opción ganadora como el diseño a fabricar y programar en las siguientes sesiones. Como la mano se compone de varias partes, cada grupo generará los ficheros necesarios para la impresión de una determinada parte, de forma acorde a las especificaciones dadas en la memoria por el grupo ganador. Además, se reparte entre los diferentes grupos el alfabeto de signos básico para que cada grupo se encargue de realizar un determinado conjunto de posiciones.

- Sesiones 08 a 11: Dedicadas a la impresión 3D de la mano robótica. Cada grupo se planifica y realiza las tareas asociadas al uso del software e impresora que les permita crear las diferentes partes de la mano robótica.

- Sesiones 11 a 15: Dedicadas a la programación de los movimientos asignados. De nuevo, el grupo se planifica y realiza las tareas de programación del microcontrolador que controla el movimiento de los diferentes servomotores que controlan la movilidad de las diferentes partes de la mano robótica. 


\section{Justificación de la necesidad del desarrollo}

Según la presentación realizada por el ponente invitado de la asociación de personas sordomudas, se estima que las personas con sordera en todo el mundo alcanzan ya la cifra de 59 millones. Solo en España existen 39.583 personas con sordera profunda; es decir, que carecen de cualquier posibilidad de escuchar. En cambio, son 1.064.100 las personas con discapacidad auditiva en España. De ellas, 303.000 se encuentran en el rango de edad entre 6 y 64 años. Se entiende por discapacidad auditiva aquella deficiencia auditiva que supone una limitación para realizar actividades diarias durante al menos un año. Muchas de estas personas utilizan dispositivos que les permite escuchar mejor. Aun así, muchos de ellos necesitan apoyarse en la lengua de signos para poder integrarse socialmente.

Según el ponente, se estima que, en España, el número total de personas sordas usuarias de la lengua de signos se encuentra entre las 70.000 y 150.000 personas. Por tanto, el número de personas con discapacidad auditiva sin acceso a esta lengua puede ser muy elevado y esto supone en muchos casos una gran limitación en la integración social de estas personas. Este dato ha sido motivante para la elección del tema del presente proyecto. La idea de este proyecto es desarrollar un sistema tal que, si se introduce por teclado una determinada letra del alfabeto, su símbolo asociado en la lengua de signos sea reproducido por la mano robótica desarrollada.

\section{Actividad de investigación}

Durante las semanas dedicadas a la búsqueda de información, los estudiantes han de trabajar en dos frentes de análisis. El primero de ellos, con un enfoque claramente tecnológico, donde deben realizar investigaciones sobre diferentes manos biónicas existentes en el mercado y pensar en formas de reproducirla. Para ello, también deben dedicar cierto tiempo a analizar desde el punto de vista fisiológico cómo funciona la mano y la biomecánica asociada a los movimientos.

La segunda parte de investigación que tuvieron que realizar fue analizar el lenguaje de signos para ver cómo se estructuraban las representaciones del alfabeto, para así proponer una estrategia de implementación de los diferentes signos. Como resultado de esta investigación se obtuvo que la lengua de signos presenta 30 configuraciones, donde 11 necesitan, además de cambiar las posiciones de los dedos, el movimiento adicional y de forma conjunta del brazo o muñeca. A fin de no complicar el diseño teniendo que diseñar un brazo adicional a la mano, se acordó que este movimiento podría ser simulado como un movimiento de la mano similar al que se hace de izquierda a derecha cuando uno saluda a alguien en la distancia. La figura 1 muestra un ejemplo de las posiciones que debe tener la mano para algunas letras del abecedario. En la izquierda se muestran ejemplos de posiciones simples y en la derecha ejemplos de letras que en la lengua de signos tienen una representación que requieren el movimiento adicional comentado. 
Figura 1. Ejemplos de posiciones de la mano en la lengua de signos. En la izquierda posiciones simples y en la derecha posiciones con movimiento adicional
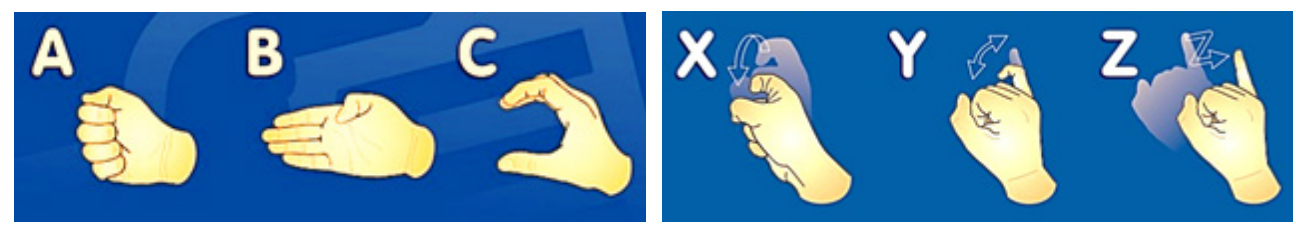

\section{Materiales disponibles}

Cada uno de los grupos constituidos ha dispuesto de un kit con el siguiente material para realizar sus propuestas:

Los trabajos se han desarrollado en un laboratorio dotado de ordenadores, con conexión a internet a fin de poder realizar sus búsquedas en la labor de investigación y con licencia de SolidWorks como software usado para la labor de modelado 3D, combinado con el programa gratuito Slic3r, para generar los ficheros G-code del modelo 3D propuesto, junto a entorno de desarrollo o IDE (Integrated Development Environment) para la programación del microcontrolador Arduino.

El aula tenía dos impresoras modelos Creality Ender 3, dotada de rollos de filamento. Se establecieron turnos en cada grupo para que tuvieran disponibilidad tanto en clase como fuera de clase para poder imprimir sus diseños.

Finalmente, cada grupo disponía también de un kit de material electrónico, constituido por una placa Arduino Mega, un conjunto de servomotores para poder dotar de movimiento a las partes móviles de la mano y un conjunto de componentes electrónicos como resistencias, condensadores, diodos LEDs, pulsadores y cables diversos para poder implementar todas las interfaces electrónicas necesarias para el control.

\section{Presentación de propuestas y fabricación de la mano.}

Los diferentes grupos presentaron cada una de sus propuestas de diseño. Se pusieron en común y se analizaron las ventajas e inconvenientes de cada una de ellas. Finalmente se realizó una votación por un diseño ganador. El diseño que obtuvo la mejor puntuación es el representado en la figura 2. 
Figura 2. Propuesta de diseño seleccionada para realizar su implementación física

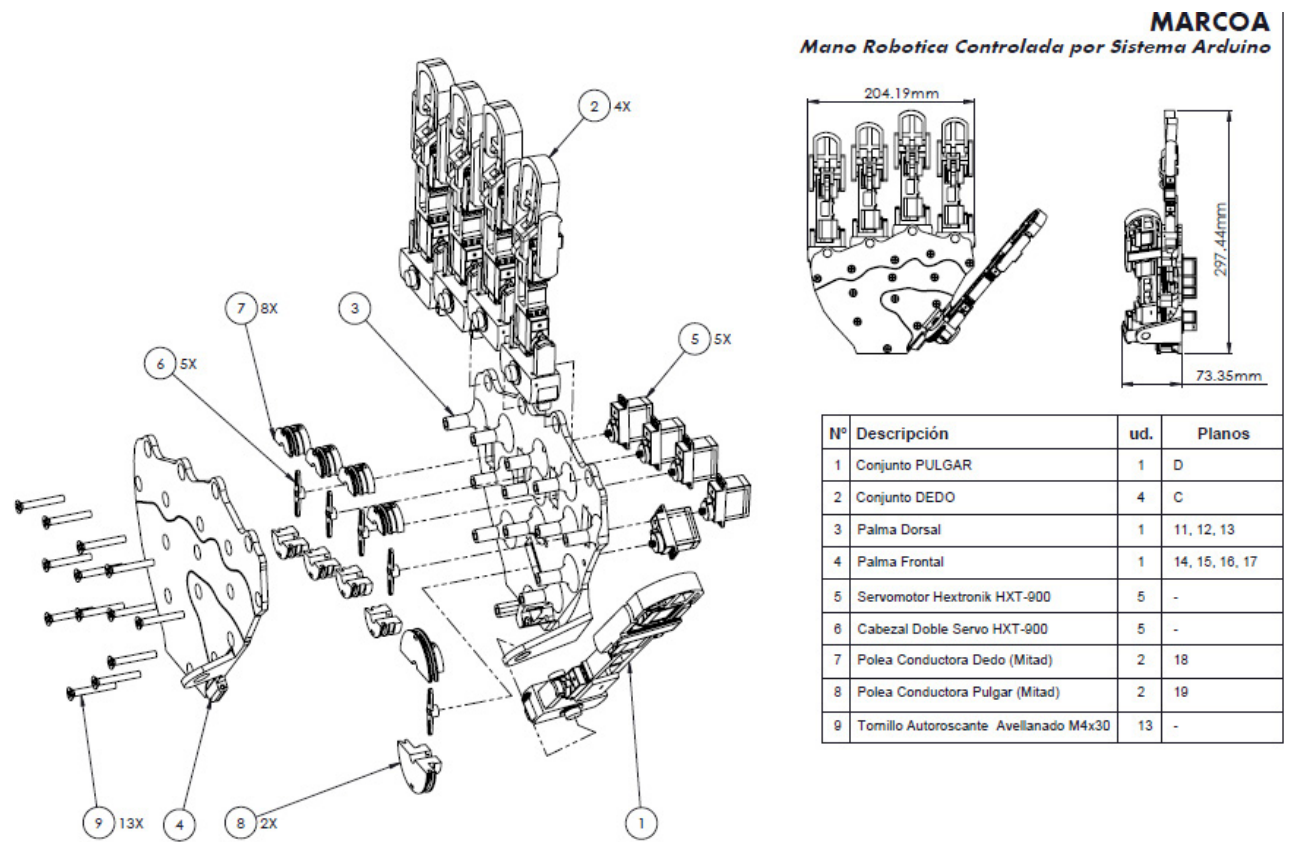

Figura 3. Modelado y fabricación 3D. a) Detalle de uno de los dedos. b) Sistema final completo

b)

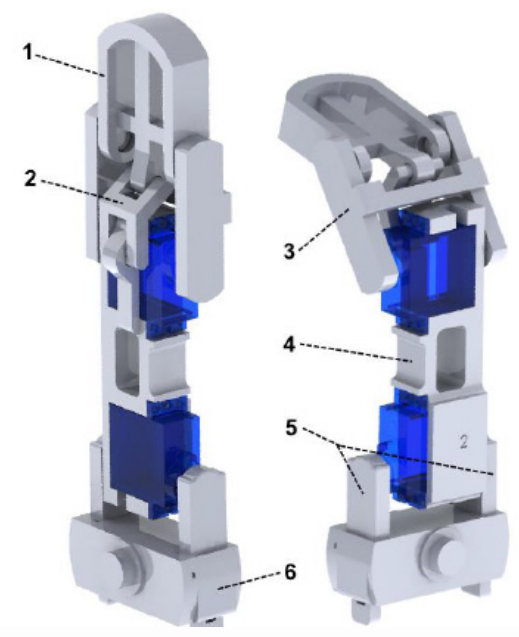

b)

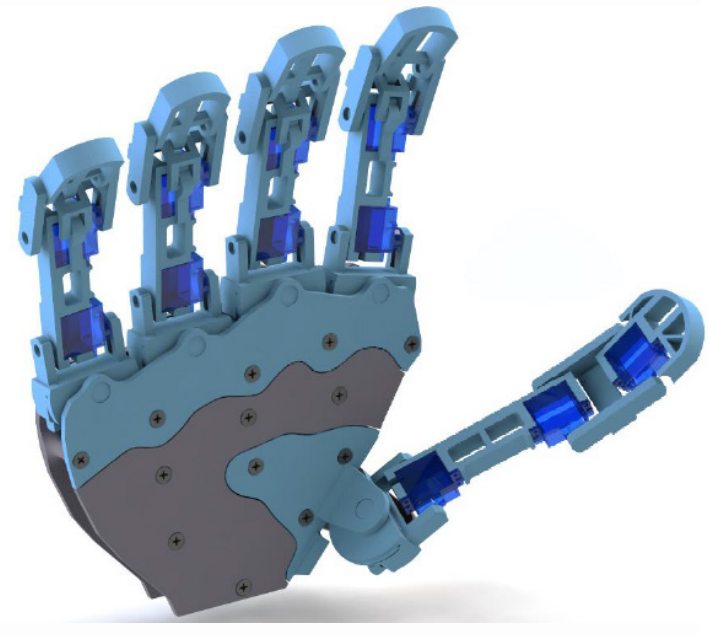


Se trata de un diseño en el que el movimiento total es controlado por 16 servomotores. 3 por cada dedo, más uno adicional que genera el movimiento adicional de mano que requieren algunas letras del alfabeto. A fin de optimizar el proceso de fabricación, dado que el trabajo incluía modelos y medidas, se repartieron las diferentes partes de la mano entre los diferentes grupos, y cada uno de ellos se dedicó a modelar e imprimir utilizando impresora $3 \mathrm{D}$ cada una de las partes desarrolladas. De esta forma, cada grupo estaba encargado de la fabricación de una parte, pero el desarrollo global era conjunto, con la unión de las diferentes partes en un prototipo final. La figura 3 pone como ejemplo el modelado y fabricación con impresora $3 \mathrm{D}$ de las diferentes partes constitutivas. La figura 3 a muestra el modelado y partes constitutivas de un dedo y la figura $3 \mathrm{~b}$ muestra el resultado de fabricación del sistema completo.

\section{Programación de los movimientos}

Una vez fabricada la mano, se repartió entre grupos la tarea de programar los diferentes movimientos. A cada grupo se le asignaron 5 movimientos: tres de ellos básicos y 2 de ellos que requirieran del movimiento adicional. En consecuencia, había movimientos asignados a más de un grupo y de esta forma se aseguraba que siempre se pudiera alcanzar que se consiguiera finalizar con éxito por parte de alguno de los grupos las tareas realizadas. La figura 4 muestra algunos resultados obtenidos tras el proceso de programación, en el que se observa la posición marcada por el lenguaje de signos asociada a la letra, junto a la reproducción que hace la programación de la mano de la misma. En la imagen se puede ver en la primera columna la letra teórica que se pretende codificar, en la segunda se observa el prototipo fabricado programado para replicar la posición y en la tercera columna se aprecia la posición adecuada de la mano, realizada por uno de los miembros de la asociación. 
Figura 4. Ejemplo de resultados tras la fase de programación

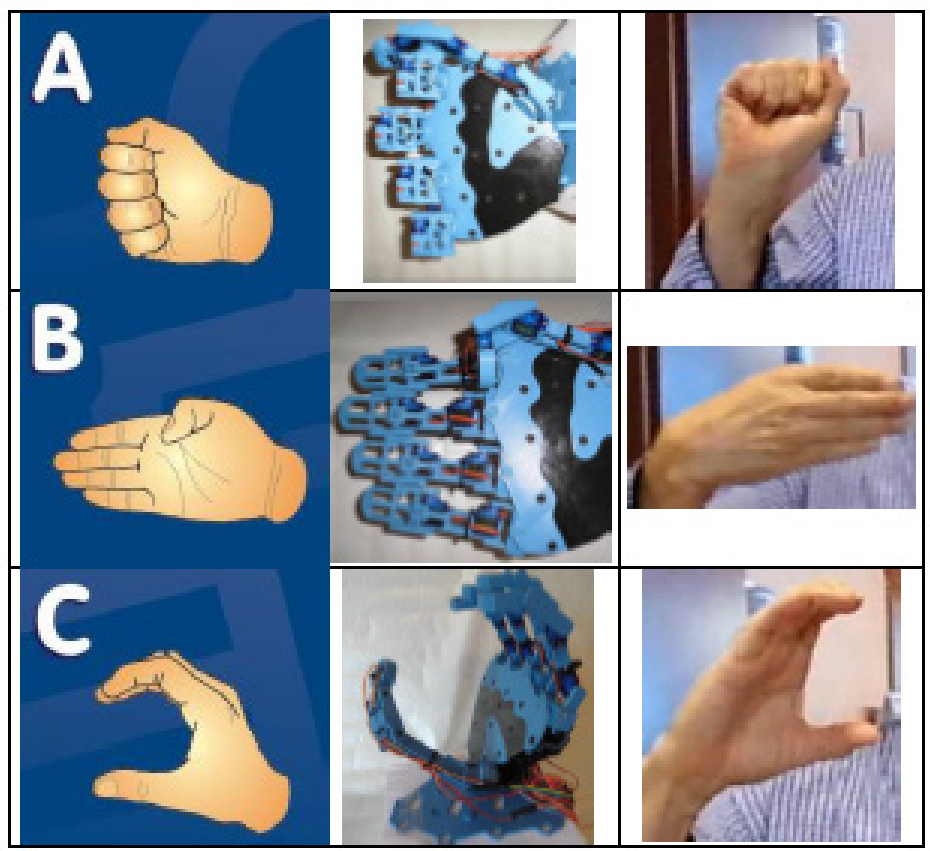

\section{RESULTADOS Y CONCLUSIONES}

Con objeto de evaluar el grado de satisfacción y aprendizaje desde el punto de vista del alumno, se procedió a realizar una pequeña encuesta a todos los estudiantes, que la realizaron de forma individual. Si bien como dato adicional se tenía la evaluación realizada por parte del profesor, se ha incluido un ítem en el que se consultaba al alumno si le había ayudado a mejorar su aprendizaje. En concreto, los ítems consultados fueron los siguientes:

- ¿Considera adecuada la organización y planificación de la experiencia? Un $54 \%$ consideró que mucho y un 31\% que bastante, por lo que se estima que la estructura de sesiones descritas en el apartado anterior ha sido bien recibida por parte del alumno.

- ¿ ¿La información recibida ha sido suficiente? Un 46\% estaba muy de acuerdo y un $38 \%$ que bastante, por lo que se interpreta que sí se ha tenido la suficiente información para el desarrollo de las tareas.

- ¿Las sesiones han sido motivadoras e interesantes? En este caso están más repartidas las respuestas. La sensación global es buena (38\% Mucho y 31\% 
bastante), pero hay un 30\% que considera que poco o nada, por lo que de cara a experiencias futuras se marca como punto de mejora el incrementar la motivación y el interés en las sesiones.

- ¿Las relaciones con los responsables ha sido satisfactoria? Este ítem intentaba valorar las tutorías realizadas. La mayor parte las encontraba satisfactorias (Mucho 62\% y Bastante un 31\%) frente a un 8\% que las habían encontrado poco satisfactorias.

- ¿Te ha beneficiado en las relaciones sociales? Parece que una gran mayoría (69\%) ha notado que la experiencia les ha sido de utilidad para mejorar esta competencia, frente un $30 \%$ que no considera que haya tenido poco (15\%) o nada (15\%) de impacto en ella.

- ¿Ha contribuido a mejorar su aprendizaje? Una vez más, una gran mayoría (Mucho 38\% y Bastante 38\%) reconoce que sí ha tenido un impacto positivo en su aprendizaje, frente a un $23 \%$ que considera que ha contribuido poco (15\%) o nada (8\%).

- ¿Consideras que la experiencia ha sido beneficiosa para la mejora de tus capacidades? Una gran parte de los estudiantes considera que ha sido beneficioso para la mejora de sus capacidades (62\% Mucho y 31\% Bastante), frente a un $8 \%$ que considera que ha sido poco beneficiosa.

- ¿El horario ha sido adecuado? La mayor parte de los estudiantes consideran acertado el horario elegido (Mucho 46\% y bastante 54\%), no habiendo gente disconforme con este punto.

La figura 5 resume gráficamente los resultados obtenidos de las valoraciones de los estudiantes de los ítems comentados. Conviene resaltar que, dado que la finalidad era solamente tener esa estimación del grado de satisfacción con la experiencia comentada, no se realizó un tratamiento estadístico profundo de la misma, en el sentido clásico de sacar intervalos de confianza, sino que se hizo un procesamiento básico de obtención de los principales valores descriptivos de los datos.

Desde el punto de vista docente, la experiencia estuvo supervisada por dos profesores, uno con experiencia en el campo del modelado $3 \mathrm{D}$ y la fabricación aditiva y otro con experiencia en el campo de la electrónica; colaborando, además, un estudiante de doctorado con 'venia docendi' y un becario de investigación que ofrecieron apoyo en la resolución de dudas y problemas durante las sesiones prácticas. Sus conclusiones sobre la experiencia se pueden resumir en los siguientes puntos:

- Se ha notado una clara evolución de los grupos. En las primeras sesiones se tenía la impresión de que los grupos se encontraban algo perdidos, quizás por no estar los alumnos acostumbrados a este tipo de experiencias, y conforme avanzaban las semanas se notaba una mejora progresiva en el funcionamiento de los mismos. 
- Al ser una asignatura cuatrimestral, la duración de sólo 15 semanas ha sido, en cierta medida, un 'handicap'. Cuando el funcionamiento de los grupos ya era óptimo acabó el cuatrimestre y tanto profesores como alumnos se han quedado con la sensación que de haber sido una asignatura anual se hubieran conseguido prototipos mucho mejores.

- La planificación de las sesiones e impartición conjunta con varios docentes ha sido crucial. El tiempo de las sesiones, sobre todo las primeras, se ha ido muchas veces en la resolución de dudas.

- Se ha observado que en los grupos en los que algún alumno ha adquirido el rol de jefe de grupo y lo ha ejercido con liderazgo se han obtenido mejores resultados que aquéllos en los que no ha habido un jefe de grupo claro. De cara a futuras repeticiones de la experiencia se propone tratar de realizar un reparto de grupos en los que haya un jefe de grupo claro con capacidad de ejercer este liderazgo.

- La experiencia global que se tiene es que, superada esa fase inicial de crisis donde los alumnos se encontraban algo perdidos y fue necesaria una mayor supervisión, el proceso de aprendizaje e implicación de los estudiantes ha sido mayor que en las clases tradicionales.

Figura 5. Resultados de la encuesta de valoración realizada

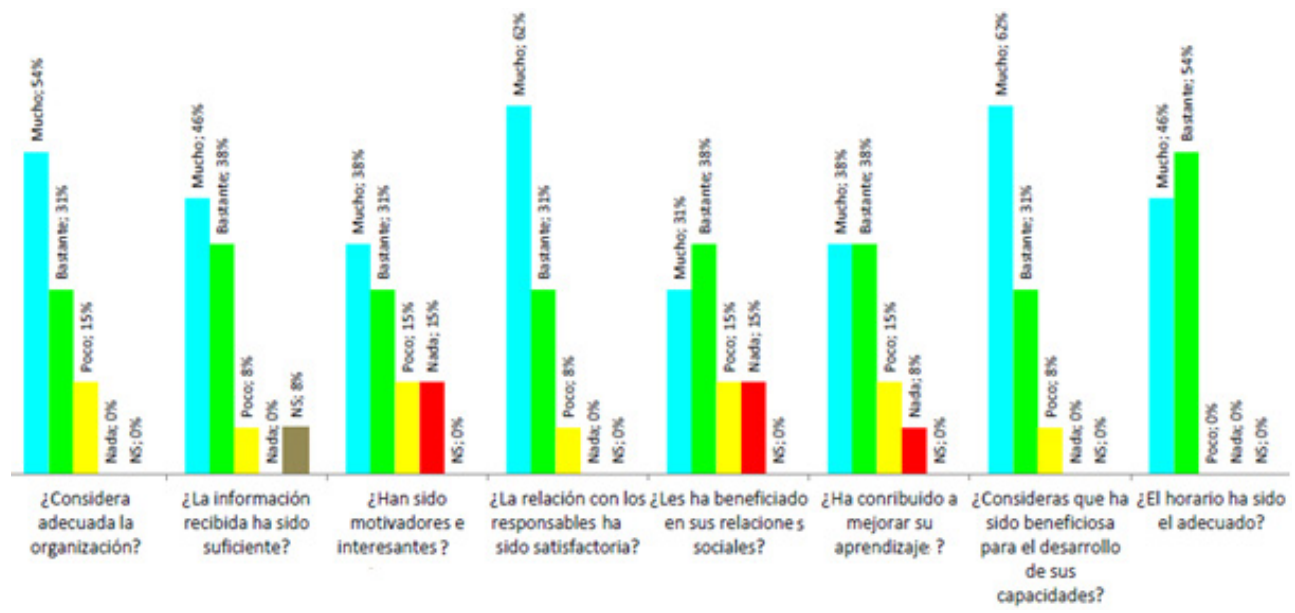

Finalmente, en cuanto a la colaboración con el integrante de la asociación, su participación en la primera sesión fue clave para poder dar una idea del contexto del marco de trabajo y justificar la necesidad del diseño. Posteriormente, también estuvo asesorando sobre las formas correctas en la que se debían posicionar las manos a la hora de ejecutar la representación de las diferentes letras del alfabeto que usaron los grupos para la implementación. Por motivos laborales no pudo asistir 
a la última sesión de presentación de resultados, pero tuvo oportunidad de ver el prototipo en una feria de ciencia en la que se expuso en un stand de la universidad. La realimentación recibida por su parte es que el prototipo resulta de interés, si bien la solución utilizada con objeto de no complicar excesivamente el diseño de usar el movimiento adicional que supliera el juego de muñeca que necesitan algunas letras del alfabeto imposibilita una aplicación real al cien por cien del prototipo, ya que si la persona que usa el prototipo no es consciente de esta peculiaridad no es capaz de interpretar lo que se codifica. Esto refuerza la idea de que esta iniciativa quizás sería más adecuada aplicarla en una asignatura anual, donde habría más semanas para poder realizar la implementación completa. Además, la lengua de signos tiene códigos propios que aglutinan palabras y conceptos, de forma que se evite tener que ir codificando uno a uno los caracteres de esas palabras, lo que abre la posibilidad de ir ampliando el repertorio de movimientos en sucesivas iteraciones de la experiencia. Por tanto, de cara a futuro, plantean varias opciones a la hora de volver a repetir la iniciativa: usar el prototipo resultante de esta experiencia para ir ampliando el repertorio de signos, o abrir una vía en la que se retomen partes del prototipo ya implementado y se vaya realizando una ampliación dotándole de muñeca y codo para poder realizar de forma fiel todo el conjunto de movimientos.

\section{REFERENCIAS}

Arratia Figueroa, A. (2008). Ética, solidaridad y aprendizaje servicio en la educación superior. Acta Bioethica, 24(1), 61-67. https://doi.org/10.4067/S1726569X2008000100008

Batle R. (2011). ¿De qué hablamos cuando hablamos de aprendizaje-servicio? Revista Crítica, 972, 49-54.

Beltrán, P., y Rodríguez, C. (2017). Modelado e impresión $3 \mathrm{D}$ en la enseñanza de las matemáticas: un estudio exploratorio. ReiDoCrea, 6(2), 16-28.

Benjamin, L. (2020). 3D printing of Anatomical Models for Veterinary Education. Canada-Caribbean Institute Journal, 1(1).

Bonet, A., Meier, C., Saorin, J. L., de la Torre, J., y Carbonell, C. (2017). Tecnologías de diseño y fabricación digital de bajo coste para el fomento de la competencia creativa. Arte, individuo y sociedad, 29(1), 89-104. https://doi.org/10.5209/ $\underline{\text { ARIS. } 51886}$
Bosqué, C. (2015). What are you printing? Ambivalent emancipation by $3 \mathrm{D}$ printing. Rapid Prototyping Journal, 21(5), 572581. https://doi.org/10.1108/RPJ-o92014-0128

Brannon, J. P., Ramírez, I., Williams, D., Barding Jr, G. A., Liu, Y., McCulloch, K. M., y Stieber, S. C. E. (2020). Teaching Crystallography by Determining Small Molecule Structures and 3-D Printing: An Inorganic Chemistry Laboratory Module. Journal of Chemical Education 97 (8), 2273-2279. https://doi.org/10.1016/j. cemconres.2018.05.006

Buswell, R. A., De Silva, W. L., Jones, S. Z., y Dirrenberger, J. (2018). 3D printing using concrete extrusion: A roadmap for research. Cement and Concrete Research, 112, 37-49.

Butin, D. W. (2003) Of what use is it? Multiple conceptualizations of service learning within education. Teachers College Record 105(9), 1674-1692. https://doi. org/10.1046/j.1467-9620.2003.00305.X 
Bull G., Haj-Hariri H., Atkins R., y Moran P. (2015). An Educational Framework for Digital Manufacturing in Schools. $3 D$ Printing and Additive Manufacturing, 2(2), 42-49. https://doi. org/10.1089/3dp.2015.0009

Cobo Romaní, C., y Moravec, J. W. (2011). Aprendizaje Invisible. Hacia una nueva ecología de la educación. Collecció Transmedia XXI. Laboratori de Mitjans Interactius / Publicacions i Edicions de la Universitat de Barcelona. Barcelona.

Dahle, R. (2018). The Use of 3-D Printing in Teaching MEMS Device Design and Fabrication, 18th International Symposium on Antenna Technology and Applied Electromagnetics (ANTEM), pp. 1-2. https://doi.org/10.1109/ ANTEM.2018.8572887

Dahle, R., Hart, S., y Hart, K. (2019). Using a 3-D Printed Mechatronics Project to Simulate MEMS Design and Fabrication. IEEE Transactions on Education 62(1), pp. 27-33. https://doi.org/10.1109/ TE.2018.2816572

Domínguez, I. A., Romero, L., Espinosa, M. M., y Domínguez, M. (2013). Impresión 3D de maquetas y prototipos en arquitectura y construcción. Revista de la construcción 12(2), 39-53. https://doi.org/10.4067/ So718-915X2013000200004

Dilling, F., y Witzke, I. (2020). The Use of 3 D-Printing Technology in Calculus Education: Concept Formation Processes of the Concept of Derivative with Printed Graphs of Functions. Digital Experiences in Mathematics Education, 1-20. https:// doi.org/10.1007/s40751-020-00062-8

Escobar, C. (2018, o1 de enero). Breve historia de la impresión 3D. Impresoras3D.com. https://goo.gl/N7U9KP

Gonzalez-Gomez, J., Valero-Gomez, A., Prieto-Moreno, A., y Abderrahim, M. (2012). A new open source 3d-printable mobile robotic platform for education. Advances in autonomous mini robots (pp. 49-62). Springer, Berlin, Heidelberg. https://doi.org/10.1007/978-3-64227482-4 8

Hansen, A. K., Langdon, T. R., Mendrin, L. W., Peters, K., Ramos, J., y Lent, D. D. (2020). Exploring the Potential of 3D-printing in Biological Education: A Review of the Literature. Integrative and Comparative Biology. https://doi. org/10.1093/icb/icaa100

Head, D. (2019). Qué es la cultura Maker y porqué queremos traerla a la educación. Medium Website. https://medium. com/@danielitohead/la-cultura-makery-por-qu\%C3\%A9-nos-interesa-tantodesde-la-educaci\%C3\%B3n-f7c6b1703fd4

Heinze, A., Basulto-Martínez, M., SuarezIbarrola, R. (2020). Impresión 3D y sus beneficios en el campo de la educación médica, entrenamiento y asesoría del paciente. Revista Española de Educación Médica 1(1), 1-8. https://doi.org/10.6018/ edumed.421221

Hull, C. W. (1984) Apparatus for production of three-dimensional objects by stereolithography. US Patent US4575330A. https://patentimages. storage.googleapis.com/5c/ao/27/ e49642dabg9cf6/US4575330.pdf

Johnson, E., y Carter, A. (2019). Defossilization: A Review of 3D Printing in Experimental Paleontology. Frontiers in Ecology and Evolution 7. https://doi. org $/ 10.3389 /$ fevo.2019.00430

Kyriakopoulos, C. (2019). 3D Printing: A Remedy to Common Misconceptions about Earthquakes. Seismological Research Letters, 90(4), 1689-1691. https://doi.org/10.1785/0220190121

Lucas Mangas, S. (2012). AprendizajeServicio como propuesta de integración curricular del Voluntariado en la Responsabilidad Social Universitaria. Actas de Jornadas sobre Responsabilidad Social. Valladolid: Universidad de Valladolid y Caja de Burgos.

Lucas Mangas. S., y Martínez Odría, A. (2012). Programa de Orientación y 
Educación Comunitaria: "Valores y Derechos Humanos para afrontar la pobreza extrema desde la responsabilidad social y el aprendizaje-servicio". Revista de orientación educacional, 50, 73-91.

Moeck, P., Destefano, P., Kaminsky, W., $\mathrm{y}$ Snyder, T. (2019). 3D printing in the context of Science, Technology, Engineering, and Mathematics education at the college/university level. Physics Education.

Moreno, N. M., Leiva, J., y López, E. (2016). Robótica, modelado 3D y realidad aumentada en educación para el desarrollo de las inteligencias múltiples. Aula de encuentro, 18(2), 158-183.

Murrey, M. (2019). Improving Geoscience Education with a Public Database of 3D-printable Crystal Models. OSR Journal of Student Research, 5, Article 279.

Mullan, H. E., y Carter, Y. (2020). Creating a $3 \mathrm{D}$ Printed Model of the Female Perineum to Supplement Traditional Cadaveric Education. The FASEB Journal, 34(S1), 1-1. https://doi.org/10.1096/ fasebj.2020.34.s1.03699

Osakwe, O., Moore, R., Divanovic, A., Del Grippo, E., Tegtmeyer, K., Madsen, N., y Taylor, M. (2019). Improving patient experience and education on congenital heart defects: the evolving role of digital heart models, $3 D$-printing and mobile application, 144(2). https://doi.org/10.1542/peds.144.2 MeetingAbstract.340

Pernaa, J., y Wiedmer, S. (2019). A systematic review of $3 \mathrm{D}$ printing in chemistry education-analysis of earlier research and educational use through technological pedagogical content knowledge framework. Chemistry Teacher International, 1. https://doi. org/10.1515/cti-2019-0005

Peterson, J. E., y Krippner, M. L. (2019). Comparisons of fidelity in the digitization and $3 \mathrm{D}$ printing of vertebrate fossils. Journal of Paleontological Techniques, 22, 1-9.

Pinger, C. W., Geiger, M. K., y Spence, D. M. (2019). Applications of $3 \mathrm{D}$-Printing for Improving Chemistry Education. Journal of Chemical Education, 97(1), 112-117. https://doi.org/10.1021/acs. jchemed.9boo588

Reichenberger, S., Lieban, D., Russo, C., y Lichtenegger, B. (2019). 3D Printing to Address Solids of Revolution at School. Bridges 2019 Conference Proceedings (pp. 493-496). Tessellations Publishing.

Reymus, M., Fotiadou, C., Kessler, A., Heck, K., Hickel, R., y Diegritz, C. (2019). 3D printed replicas for endodontic education. International endodontic journal, 52(1), 123-130. https://doi.org/10.1111/ iej.12964

Universia. (2019). Imprimir 3D: profesión de futuro. Universia Website. https:// noticias.universia.es/practicas-empleo/ noticia/2019/12/19/1167429/imprimir3d-profesion-futuro.html

Yu, J., Han, S. D., Tang, W. N., y Rus, D. (2017). A portable, 3D-printing enabled multi-vehicle platform for robotics research and education. 2017 IEEE International Conference on Robotics and Automation (ICRA) (pp. 14751480). IEEE. https://doi.org/10.1109/ ICRA.2017.7989176

\section{PERFIL ACADÉMICO Y PROFESIONAL DE LOS AUTORES}

Juan Suardíaz Muro. Doctor Ingeniero Industrial. Profesor Titular en el Departamento de Automática, Ingeniería Eléctrica y Tecnología Electrónica de la Universidad Politécnica de Cartagena. Tiene concedidos tres sexenios de 
investigación. Sus líneas de investigación se centran en el desarrollo de sistemas de automatización y control, el diseño electrónico orientado a aplicaciones en el Internet de las cosas y la innovación docente en el campo de la enseñanza de la electrónica en carreras de ingeniería. ID: https://orcid.org/0000-0003-0648-2541

e-mail: juan.suardiaz@upct.es

Dirección:

Dpto. de Automática, Ingeniería Eléctrica y Tecnología Electrónica.

Universidad Politécnica de Cartagena.

Campus Muralla del Mar

30202 Cartagena (España).

Mario Pérez-Gomariz. Doctorando en el Departamento de Tecnologías de la Información y la Comunicación de la Escuela Técnica Superior de Ingeniería de Telecomunicación de la Universidad Politécnica de Cartagena. Sus líneas de investigación se centran en el campo de la inteligencia artificial, el aprendizaje automático y minería de datos en el sector industrial y el campo de la innovación docente en enseñanzas relacionadas con el ámbito de la ingeniería de telecomunicaciones. ID: https://orcid.org/0000-0003-1099-5219

E-mail: mario.perez@edu.upct.es

Dirección:

Grupo de Investigación DINTEL. Edificio ELDI

Universidad Politécnica de Cartagena.

Campus Muralla del Mar

30202 Cartagena (España).

Andrés Cabrera Lozoya. Doctor Ingeniero de Telecomunicación e Ingeniero Técnico Industrial. Investigador en la División de Innovación en Sistemas Telemáticos y Tecnología Electrónica (DINTEL) de la Universidad Politécnica de Cartagena (UPCT), España. Sus líneas de investigación se centran en el desarrollo de herramientas innovadoras de aprendizaje web, con el foco en desarrollos móviles y la mejora de la experiencia de usuario.

E-mail: andres.cabrera@upct.es

Dirección:

Dpto. de Física Aplicada y Tecnología Naval.

Universidad Politécnica de Cartagena.

Campus Muralla del Mar

30202 Cartagena (España). 
René Ove Do Carmo Trolle. Graduado en Ingeniería Mecánica. Becario de investigación en el grupo División de Innovación en Sistemas Telemáticos y Tecnología Electrónica (DINTEL) de la Universidad Politécnica de Cartagena (UPCT), España. Sus líneas de investigación se centran en el campo del modelado 3D y la fabricación aditiva, teniendo amplia experiencia en el campo de la impresión 3D. E-mail: rene.trolle@edu.upct.es

Dirección:

Grupo de Investigación DINTEL. Edificio ELDI

Universidad Politécnica de Cartagena.

Campus Muralla del Mar

30202 Cartagena (España).

Fecha de recepción del artículo: 29/05/2020

Fecha de aceptación del artículo: 04/07/2020

Fecha de aprobación para maquetación: 21/09/2020 